\title{
Prevalência de sintomas de ansiedade e depressão em pessoas com a Síndrome do Túnel do Carpo*
}

\section{Prevalence of Anxiety and Depression Symptoms in People with Carpal Tunnel Syndrome}

\author{
Henver Ribeiro Paiva Filho ${ }^{1}$ Franco Luiz Cabral Pedroso ${ }^{2}$ Fernando Brito Bueno ${ }^{2}$ \\ Valdênia Graças Nascimento Paiva ${ }^{10}$ Elias Felix Oliveira ${ }^{1}$ Murilo Antônio Rocha ${ }^{2}$ \\ 1 Universidade Federal do Triângulo Mineiro, Uberaba, MG, Brasil \\ 2 Serviço de Ortopedia e Traumatologia, Universidade Federal do \\ Triângulo Mineiro, Uberaba, MG, Brasil \\ Endereço para correspondência Valdênia Graças Nascimento Paiva, MD, \\ Ambulatório Maria da Clória - Ortopedia e Traumatologia, Hospital de \\ Clínicas da Universidade Federal do Triângulo Mineiro (HC-UFTM/Filial \\ EBSERH), Uberaba, MG, Brasil (e-mail: vallfmtm@yahoo.com.br).
}

Rev Bras Ortop 2020;55(4):438-444.

\section{Resumo \\ Palavras-chave \\ - síndrome do túnel do carpo \\ - ansiedade \\ - depressão}

Abstract
Objetivos Demonstrar a prevalência de sintomas de depressão e ansiedade em pessoas com a síndrome do túnel do carpo atendidas em um ambulatório de cirurgia da mão e descrever as características clínicas e epidemiológicas dessa população.

Métodos Foram avaliadas pessoas com o diagnóstico de síndrome do túnel do carpo na consulta inicial em um período de coleta de dados de 6 meses. Características clínicas e epidemiológicas foram anotadas, e as pessoas que possuíam diagnóstico de ansiedade e/ou depressão foram avaliadas.

Resultados Um total de 101 pessoas apresentavam síndrome do túnel do carpo e destas, 38 apresentavam diagnóstico de depressão e 29 de ansiedade. Houve prevalência de mulheres de baixa renda, com predominância do nível de escolaridade fundamental. Mais da metade dos pacientes apresentava pelo menos uma comorbidade sistêmica associada.

Conclusão Tanto para ansiedade como para depressão, as características que influenciaram estatisticamente nos sintomas em pacientes com síndrome do túnel do carpo independente das demais características avaliadas foram gênero, tabagismo, e renda familiar $(p<0,05)$.

Objectives To demonstrate the prevalence of depression and anxiety symptoms in patients with carpal tunnel syndrome treated at a hand surgery outpatient clinic and to describe the clinical and epidemiological characteristics of this population.

Methods People diagnosed with carpal tunnel syndrome at the initial visit were evaluated over a 6-month period for data collection. Clinical and epidemiological characteristics were noted, and patients diagnosed with anxiety and/or depression were evaluated.

\footnotetext{
* Trabalho feito no Serviço de Ortopedia e Traumatologia, Universidade Federal do Triângulo Mineiro, Uberaba, Minas Gerais, Brasil.
}

recebido

14 de Junho de 2019

aceito

15 de Agosto de 2019
DOI https://doi.org/

10.1055/s-0039-3400517. ISSN 0102-3616.
Copyright $\odot 2020$ by Sociedade Brasileira License terms de Ortopedia e Traumatologia. Published by Thieme Revinter Publicações Ltda, Rio de Janeiro, Brazil 
Results In total, 101 people had carpal tunnel syndrome, including 38 diagnosed with

Keywords

- carpal tunnel syndrome

- anxiety

- depression depression and 29 with anxiety. Most patients were low-income women, with elementary school-level education. More than half of the patients had at least one associated systemic comorbidity.

Conclusion Independent characteristics that statistically influenced anxiety and depression symptoms in patients with carpal tunnel syndrome were gender, smoking and family income $(p<0.05)$.

\section{Introdução}

A síndrome do túnel do carpo (STC) é considerada a neuropatia periférica mais comum. É caracterizada pela presença de sintomas de dormência e formigamento devido à compressão do nervo mediano ao nível do túnel do carpo, com prevalência estimada em $3 \%$ da população geral. ${ }^{1}$ A depressão está entre os problemas de saúde mental mais comuns, afetando em torno de 300 milhões de pessoas em todo o mundo. ${ }^{2}$ É considerada uma das principais causas de incapacidade, com perdas substanciais de produtividade no trabalho e grande impacto financeiro à sociedade. ${ }^{3}$

Na STC, a relação entre sintomas depressivos e os resultados cirúrgicos é de particular interesse, visto que ambos são condições altamente prevalentes, especialmente em mulheres. ${ }^{4}$

Evidências sustentam o papel significativo dos estilos de vida como determinantes de depressão, dentre eles a atividade física, o consumo de álcool, o tabagismo, e a obesidade., ${ }^{5,6}$

O diagnóstico de depressão é realizado por meio da escuta às queixas do paciente e da busca ativa por sintomas que possam ser negligenciados ou não verbalizados. Dentro desse espectro, as classificações diagnósticas (Classificação Internacional de Doenças e Problemas Relacionados à Saúde - CID 10 e o Manual Diagnóstico e Estatístico de Transtornos Mentais DSM-5) definem categorias nosológicas a partir de parâmetros clínicos como duração, persistência, abrangência, perturbação do funcionamento psicológico e fisiológico, e desproporção em relação a um fator desencadeante. Em alguns países desenvolvidos, o número de pessoas que sofrem de depressão tem aumentado. No Japão, que é considerado um dos países com maior índice de suicídio do mundo, a depressão é a principal causa de afastamento do trabalho dentre os trabalhadores em atividade. ${ }^{7}$ Estudos prévios demonstram que condições como depressão e ansiedade podem contribuir para a limitação funcional em pacientes com enfermidades musculoesqueléticas na extremidade superior. ${ }^{8}$

É primordial a compreensão sobre fatores epidemiológicos, sociais, e culturais das pessoas com STC e afecções relacionadas à saúde mental, especificamente a ansiedade e a depressão, 0 que pode otimizar o cuidado do paciente como um todo. 0 nosso trabalho se justifica pela escassez de estudos publicados sobre essa temática.

O objetivo do presente estudo foi demonstrar as características clínicas, epidemiológicas, e socioeconômicas das pessoas com a STC em acompanhamento ambulatorial que apresentam sintomas de depressão e ansiedade.

\section{Métodos}

Foi realizado estudo observacional, descritivo, transversal, quali-quantitativo, com avaliação de pacientes em acompanhamento ambulatorial para a STC, agendados de forma consecutiva no período de 6 meses. Todos os procedimentos foram realizados de acordo com os padrões éticos determinados pelo Comitê de Ética em Pesquia para pesquisa em seres humanos e com a Declaração de Helsinque de 1964. O termo de consentimento livre e esclarecido foi obtido de todos os participantes do estudo mediante assinatura de termo específico.

Os critérios de inclusão foram pessoas de ambos os gêneros, acima de 18 anos de idade, que apresentavam diagnóstico clínico de STC em acompanhamento ambulatorial na especialidade cirurgia da mão e diagnóstico clínico de depressão e/ou ansiedade em acompanhamento ambulatorial na especialidade psiquiatria. Foram excluídas pessoas portadoras de outras doenças neuropsiquiátricas e aquelas com limitações cognitivas que impossibilitassem responder aos questionamentos.

Durante $o$ atendimento ambulatorial os participantes foram interrogados sobre sintomas de ansiedade e depressão, conforme o protocolo de atendimento ambulatorial, no qual continha variáveis clínicas e epidemiológicas (idade, gênero, índice de massa corporal, estado civil, número de filhos, tabagismo, escolaridade, doenças associadas, profissão, renda familiar, e tempo de diagnóstico) e questionário baseado nos critérios de ansiedade e depressão segundo o DSM $-5 .{ }^{2}$

As características qualitativas foram descritas com uso de frequências absolutas e relativas, e as idades com uso de média e desvio padrão em todos os pacientes avaliados. A prevalência de ansiedade e depressão foi demonstrada segundo cada característica qualitativa e verificada a associação com uso de testes qui-quadrado ou testes exatos (teste exato de Fisher ou teste da razão de verossimilhanças). As idades foram descritas segundo ansiedade e depressão e comparadas com uso do teste t-Student. ${ }^{9}$ Foram estimadas as razões de chances de cada variável avaliada para ansiedade e depressão com os respectivos intervalos com $95 \%$ de confiança com uso de regressão logística simples. $^{10}$

Modelos de regressão logística múltipla ${ }^{10}$ foram estimados para ansiedade e depressão, selecionando-se as variáveis que nos testes bivariados apresentaram níveis de 
significância inferiores a $0,10(p<0,10)$ e variáveis clinicamente importantes para ansiedade e depressão. Para realização das análises foi utilizado o software SPSS for Windows versão 22.0 (IBM Corp., Armon, NY, EUA) e para tabulação dos dados foi utilizado o software Microsoft Excel 2003 (Microsoft Corp., Redmond, WA, EUA). Os testes foram realizados com nível de significância de $5 \%$.

\section{Resultados}

A - Tabela 1 sintetiza as características clínicas e epidemiológicas da nossa amostra. Foram atendidas 101 pessoas com

Tabela 1 Descrição das características dos pacientes com a síndrome do túnel do carpo

\begin{tabular}{|c|c|}
\hline Variável & $\begin{array}{l}\text { Descrição } \\
(\mathrm{N}=101)\end{array}$ \\
\hline Idade (anos), mean \pm desvio padrão & $54,7 \pm 108$ \\
\hline \multicolumn{2}{|l|}{ Gênero, n (\%) } \\
\hline Feminino & $86(85,1)$ \\
\hline Masculino & $15(14,9)$ \\
\hline \multicolumn{2}{|l|}{ Índice de massa corpórea, n (\%) } \\
\hline Sobrepeso & $36(35,6)$ \\
\hline Obesidade & $31(30,7)$ \\
\hline Normal & $34(33,7)$ \\
\hline \multicolumn{2}{|l|}{ Estado civil, n (\%) } \\
\hline Solteiro & $15(14,9)$ \\
\hline Casado & $67(66,3)$ \\
\hline Divorciado & $13(12,9)$ \\
\hline Viúvo & $6(5,9)$ \\
\hline \multicolumn{2}{|l|}{ Filhos, n (\%) } \\
\hline $\operatorname{sim}$ & $94(93,1)$ \\
\hline Não & $7(6,9)$ \\
\hline \multicolumn{2}{|l|}{ Tabagismo, n (\%) } \\
\hline Não & $69(68,3)$ \\
\hline $\operatorname{sim}$ & $16(15,8)$ \\
\hline Ex-tabagista & $16(15,8)$ \\
\hline \multicolumn{2}{|l|}{ Escolaridade, n (\%) } \\
\hline Alfabetizado & $24(23,8)$ \\
\hline Ensino fundamental incompleto & $28(27,7)$ \\
\hline Ensino fundamental completo & $18(17,8)$ \\
\hline Ensino médio incompleto & $5(5)$ \\
\hline Ensino médio completo & $21(20,8)$ \\
\hline Ensino superior & $5(5)$ \\
\hline \multicolumn{2}{|l|}{ Outras doenças, n (\%) } \\
\hline Uma comorbidade sistêmica & $31(30,7)$ \\
\hline Duas ou mais comorbidades sistêmicas & $39(38,6)$ \\
\hline Sem comorbidades sistêmicas & $31(30,7)$ \\
\hline \multicolumn{2}{|l|}{ Profissão, n (\%) } \\
\hline Aposentado/Do lar & $40(39,6)$ \\
\hline
\end{tabular}

Tabela 1 (Continued)

\begin{tabular}{|l|l|}
\hline Em atividade laboral & $61(60,4)$ \\
\hline Renda familiar, n (\%) & \\
\hline Até 1 salário & $76(75,2)$ \\
\hline Entre 1 e 3 salários & $22(21,8)$ \\
\hline Maior que 3 salários & $3(3)$ \\
\hline Diagnóstico de depressão, n (\%) & \\
\hline Depressão & $18(47,4)$ \\
\hline Ansiedade & $8(21,1)$ \\
\hline Misto - depressão e ansiedade & $12(31,6)$ \\
\hline Tempo de diagnóstioc, $\mathrm{n}(\%)^{*}$ & \\
\hline 1 ano & $5(13,2)$ \\
\hline Entre 1 e 5 anos & $17(44,7)$ \\
\hline Maior que 5 anos & $16(42,1)$ \\
\hline História familiar, $\mathrm{n}(\%)^{*}$ & \\
\hline Sim & $12(31,6)$ \\
\hline Não & $26(68,4)$ \\
\hline Ansiedade, n (\%) & \\
\hline Não & $72(71,3)$ \\
\hline Sim & $29(28,7)$ \\
\hline Depressão, $\mathrm{n}$ (\%) & $38(37,6)$ \\
\hline Não & \\
\hline Sim & \\
\hline
\end{tabular}

*Apenas com ansiedade e/ou depressão.

diagnóstico da STC, sendo 86 mulheres (85,1\%) e 15 homens (14,9\%). A média de idade foi 54,7 anos $\pm 10,8$ (mínimo 35 e máximo 78 anos). Obesidade foi verificada em 31 casos $(30,7 \%)$ e sobrepeso em $36(35,6 \%) .67$ pessoas $(66,3 \%)$ se declararam casadas, 15 (14,9\%) solteiras, $13(12,9 \%)$ divorciadas e $6(5,9 \%)$ viúvas. Um total de $93,1 \%$ das pessoas (94 casos) relataram possuir filhos. Tabagistas e ex-tabagistas foram igualmente relatados em 16 casos cada (15,8\%). O nível de escolaridade predominante foi o ensino fundamental (46 casos). 31 casos (30,7\%) não apresentavam comorbidades associadas, enquanto 31 (30,7\%) apresentavam somente uma doença sistêmica (hipertensão ou diabetes) e 39 (38,6\%) 2 ou mais (pelo menos hipertensão e diabetes). Um total de 61 pessoas $(60,4 \%)$ relataram estar em atividade laboral atual, enquanto 40 pessoas $(39,6 \%)$ se declararam aposentadas. A renda familiar igual ou inferior a 1 salário mínimo foi registrada em 76 pessoas (75,2\%), enquanto 3 pessoas (3\%) informaram renda familiar igual ou superior a 3 salários mínimos. A depressão foi diagnosticada em 38 casos (37,6\%) e a ansiedade em 29 (28,7\%).

A - Tabela 2 descreve as características das pessoas com diagnóstico de STC e ansiedade e a - Tabela $\mathbf{3}$ as características das pessoas com diagnóstico de STC e depressão. A - Tabela 4 demonstra os resultados dos modelos para explicar a freqüência de ansiedade e depressão ajustados pelas características pessoais e clínicas avaliadas. 
Tabela 2 Descrição da frequência de ansiedade em pacientes com síndrome do túnel do carpo segundo características pessoais e clínicas e resultado das análises não ajustadas

\begin{tabular}{|c|c|c|c|c|c|c|}
\hline \multirow[t]{2}{*}{ Variável } & \multicolumn{2}{|l|}{ Ansiedade } & \multirow[t]{2}{*}{ Razão de chances } & \multicolumn{2}{|c|}{$\begin{array}{l}\text { Intervalo de } \\
\text { confiança (95\%) }\end{array}$} & \multirow[t]{2}{*}{ valor-p } \\
\hline & Não ( $N=72)$ & $\operatorname{Sim}(N=29)$ & & Inferior & Superior & \\
\hline $\begin{array}{l}\text { Idade (anos), } \\
\text { média } \pm \text { desvio padrão }\end{array}$ & $55,4 \pm 11,5$ & $53,1 \pm 8,7$ & 0,98 & 0,94 & 1,02 & $0,349^{* *}$ \\
\hline Gênero, n (\%) & & & & & & $0,061^{*}$ \\
\hline Feminino & $58(67,4)$ & $28(32,6)$ & 6,76 & 0,85 & 54,01 & \\
\hline Masculino & $14(93,3)$ & $1(6,7)$ & 1,00 & & & \\
\hline $\begin{array}{l}\text { Índice de massa } \\
\text { corpórea, n (\%) }\end{array}$ & & & & & & 0,663 \\
\hline Sobrepeso & $24(66,7)$ & $12(33,3)$ & 1,63 & 0,57 & 4,66 & \\
\hline Obesidade & $22(71)$ & $9(29)$ & 1,33 & 0,44 & 4,03 & \\
\hline Normal & $26(76,5)$ & $8(23,5)$ & 1,00 & & & \\
\hline Estado civil, n (\%) & & & & & & $0,123 \#$ \\
\hline Solteiro & $8(53,3)$ & $7(46,7)$ & 1,00 & & & \\
\hline Casado & $52(77,6)$ & $15(22,4)$ & 0,33 & 0,10 & 1,06 & \\
\hline Divorciado & $7(53,8)$ & $6(46,2)$ & 0,98 & 0,22 & 4,34 & \\
\hline Viúvo & $5(83,3)$ & $1(16,7)$ & 0,23 & 0,02 & 2,46 & \\
\hline Filhos, n (\%) & & & & & & $0,406^{*}$ \\
\hline Sim & $68(72,3)$ & $26(27,7)$ & 0,51 & 0,11 & 2,44 & \\
\hline Não & $4(57,1)$ & $3(42,9)$ & 1,00 & & & \\
\hline Tabagismo, n (\%) & & & & & & $0,035 \#$ \\
\hline Não & $52(75,4)$ & $17(24,6)$ & 1,00 & & & \\
\hline Sim & $13(81,3)$ & $3(18,8)$ & 0,71 & 0,18 & 2,78 & \\
\hline Ex-tabagista & $7(43,8)$ & $9(56,3)$ & 3,93 & 1,27 & 12,17 & \\
\hline Escolaridade, n (\%) & & & & & & $0,602 \#$ \\
\hline Alfabetizado & $18(75)$ & $6(25)$ & 1,00 & & & \\
\hline Ensino fundamental incompleto & $22(78,6)$ & $6(21,4)$ & 0,82 & 0,23 & 2,98 & \\
\hline Ensino fundamental completo & $12(66,7)$ & $6(33,3)$ & 1,50 & 0,39 & 5,77 & \\
\hline Ensino médio incompleto & $2(40)$ & $3(60)$ & 4,50 & 0,60 & 33,71 & \\
\hline Ensino médio completo & $14(66,7)$ & $7(33,3)$ & 1,50 & 0,41 & 5,48 & \\
\hline Ensino superior & $4(80)$ & $1(20)$ & 0,75 & 0,07 & 8,09 & \\
\hline Outras doenças, n (\%) & & & & & & 0,315 \\
\hline Uma comorbidade sistêmica & $25(80,6)$ & $6(19,4)$ & 0,59 & 0,18 & 1,91 & \\
\hline $\begin{array}{l}\text { Duas ou mais } \\
\text { comorbidades sistêmicas }\end{array}$ & $25(64,1)$ & $14(35,9)$ & 1,37 & 0,50 & 3,78 & \\
\hline Sem comorbidades sistêmicas & $22(71)$ & $9(29)$ & 1,00 & & & \\
\hline Profissão, n (\%) & & & & & & 0,264 \\
\hline Aposentado/Do lar & $31(77,5)$ & $9(22,5)$ & 1,00 & & & \\
\hline Em atividade laboral & $41(67,2)$ & $20(32,8)$ & 1,68 & 0,67 & 4,19 & \\
\hline Renda familiar, n (\%) & & & & & & $0,011 \#$ \\
\hline Até 1 salário & $58(76,3)$ & $18(23,7)$ & 1,00 & & & \\
\hline Entre 1 e 3 salários & $14(63,6)$ & $8(36,4)$ & 1,84 & 0,67 & 5,09 & \\
\hline Maior que 3 salários & $0(0)$ & $3(100)$ & $\&$ & & & \\
\hline
\end{tabular}

Teste de qui-quadrado; *Teste exato de Fisher; \# Teste da razão de verossimilhanças; ${ }^{* *}$ Teste T de Student; \& Não há casos para estimar. 
442 Prevalência de Sintomas de Ansiedade e Depressão em Pessoas com a Síndrome do Túnel do Carpo Paiva Filho et al.

Tabela 3 Descrição da frequência de depressão em pacientes com síndrome do túnel do carpo segundo características pessoais e clínicas e resultado das análises não ajustadas

\begin{tabular}{|c|c|c|c|c|c|c|}
\hline \multirow[t]{2}{*}{ Variável } & \multicolumn{2}{|l|}{ Depressão } & \multirow[t]{2}{*}{ Razão de chances } & \multicolumn{2}{|c|}{$\begin{array}{l}\text { Intervalo de } \\
\text { confiança (95\%) }\end{array}$} & \multirow[t]{2}{*}{ valor $=p$} \\
\hline & Não (N=63) & $\operatorname{Sim}(N=38)$ & & Inferior & Superior & \\
\hline $\begin{array}{l}\text { Idade (anos), } \\
\text { média } \pm \text { desvio padrão }\end{array}$ & $55,1 \pm 11,3$ & $54,1 \pm 10,1$ & 0,99 & 0,96 & 1,03 & $0,653^{* *}$ \\
\hline Gênero, n (\%) & & & & & & 0,127 \\
\hline Feminino & $51(59,3)$ & $35(40,7)$ & 2,75 & 0,72 & 10,45 & \\
\hline Masculino & $12(80)$ & $3(20)$ & 1,00 & & & \\
\hline $\begin{array}{l}\text { Índice de massa } \\
\text { corpórea, n (\%) }\end{array}$ & & & & & & 0,294 \\
\hline Sobrepeso & $19(52,8)$ & $17(47,2)$ & 2,15 & 0,80 & 5,76 & \\
\hline Obesidade & $20(64,5)$ & $11(35,5)$ & 1,32 & 0,47 & 3,74 & \\
\hline Normal & $24(70,6)$ & $10(29,4)$ & 1,00 & & & \\
\hline Estado civil, n (\%) & & & & & & $0,198 \#$ \\
\hline Solteiro & $6(40)$ & $9(60)$ & 1,00 & & & \\
\hline Casado & $46(68,7)$ & $21(31,3)$ & 0,30 & 0,10 & 0,97 & \\
\hline Divorciado & $7(53,8)$ & $6(46,2)$ & 0,57 & 0,13 & 2,57 & \\
\hline Viúvo & $4(66,7)$ & $2(33,3)$ & 0,33 & 0,05 & 2,43 & \\
\hline Filhos, n (\%) & & & & & & $0,100^{*}$ \\
\hline Sim & $61(64,9)$ & $33(35,1)$ & 0,22 & 0,04 & 1,18 & \\
\hline Não & $2(28,6)$ & $5(71,4)$ & 1,00 & & & \\
\hline Tabagismo, n (\%) & & & & & & 0,075 \\
\hline Não & $47(68,1)$ & $22(31,9)$ & 1,00 & & & \\
\hline Sim & $10(62,5)$ & $6(37,5)$ & 1,28 & 0,41 & 3,98 & \\
\hline Ex-tabagista & $6(37,5)$ & $10(62,5)$ & 3,56 & 1,15 & 11,04 & \\
\hline Escolaridade, n (\%) & & & & & & $0,791 \#$ \\
\hline Alfabetizado & $15(62,5)$ & $9(37,5)$ & 1,00 & & & \\
\hline Ensino fundamental incompleto & $18(64,3)$ & $10(35,7)$ & 0,93 & 0,30 & 2,87 & \\
\hline Ensino fundamental completo & $12(66,7)$ & $6(33,3)$ & 0,83 & 0,23 & 3,00 & \\
\hline Ensino médio incompleto & $2(40)$ & $3(60)$ & 2,50 & 0,35 & 17,94 & \\
\hline Ensino médio completo & $14(66,7)$ & $7(33,3)$ & 0,83 & 0,24 & 2,84 & \\
\hline Ensino superior & $2(40)$ & $3(60)$ & 2,50 & 0,35 & 17,94 & \\
\hline Outras doenças, n (\%) & & & & & & 0,021 \\
\hline Uma comorbidade sistêmica & $24(77,4)$ & $7(22,6)$ & 0,61 & 0,20 & 1,90 & \\
\hline $\begin{array}{l}\text { Duas ou mais comorbidades } \\
\text { sistêmicas }\end{array}$ & $18(46,2)$ & $21(53,8)$ & 2,45 & 0,92 & 6,54 & \\
\hline Sem comorbidades sistêmicas & $21(67,7)$ & $10(32,3)$ & 1,00 & & & \\
\hline Profissão, n (\%) & & & & & & 0,389 \\
\hline Aposentado/Do lar & $27(67,5)$ & $13(32,5)$ & 1,00 & & & \\
\hline Em atividade laboral & $36(59)$ & $25(41)$ & 1,44 & 0,63 & 3,33 & \\
\hline Renda familiar, n (\%) & & & & & & $0,028 \#$ \\
\hline Até 1 salário & $51(67,1)$ & $25(32,9)$ & 1,00 & & & \\
\hline Entre 1 e 3 salários & $12(54,5)$ & $10(45,5)$ & 1,70 & 0,65 & 4,47 & \\
\hline Maior que 3 salários & $0(0)$ & $3(100)$ & $\&$ & & & \\
\hline
\end{tabular}

Teste do qui quadrado; *Teste exato de Fisher; \# Teste da razão de verossimilhanças; ${ }^{* *}$ Teste T de Student; \& Não há casos para estimar. 
Tabela 4 Resultado dos modelos para explicar a frequência de ansiedade e depressão ajustados pelas características pessoais e clínicas avaliadas

\begin{tabular}{|c|c|c|c|c|c|}
\hline \multirow[t]{2}{*}{ Desfecho } & \multirow[t]{2}{*}{ Variável } & \multirow[t]{2}{*}{ Razão de chnaces } & \multicolumn{2}{|c|}{$\begin{array}{l}\text { Intervalo de } \\
\text { confiança (95\%) }\end{array}$} & \multirow[t]{2}{*}{$p$} \\
\hline & & & Inferior & Superior & \\
\hline \multirow[t]{6}{*}{ Ansiedade } & Idade (anos) & 0,99 & 0,94 & 1,03 & 0,547 \\
\hline & Gênero (feminino) & 18,55 & 1,65 & 208,27 & 0,018 \\
\hline & 'Tabagismo (referência: não)' & 1,00 & & & \\
\hline & Tabagista & 0,73 & 0,16 & 3,26 & 0,676 \\
\hline & Ex-tabagista & 6,05 & 1,64 & 22,34 & 0,007 \\
\hline & Renda familiar & 5,18 & 1,75 & 15,37 & 0,003 \\
\hline \multirow[t]{10}{*}{ Depressão } & Idade (anos) & 0,99 & 0,64 & 1,05 & 0,782 \\
\hline & Gênero (feminino) & 8,91 & 1,32 & 60,29 & 0,025 \\
\hline & Filhos & 0,18 & 0,02 & 1,34 & 0,094 \\
\hline & Tabagismo (Referência: Não) & 1,00 & & & \\
\hline & Tabagista & 1,77 & 0,49 & 6,40 & 0,387 \\
\hline & Ex-tabagista & 4,30 & 1,15 & 16,13 & 0,031 \\
\hline & Renda familiar & 4,26 & 1,41 & 12,82 & 0,010 \\
\hline & Outras doenças (Referência: Sem comorbidades sistêmicas) & 1,00 & & & \\
\hline & Uma comorbidade sistêmica & 0,70 & 0,19 & 2,62 & 0,595 \\
\hline & Duas ou mais comorbidades sistêmicas & 3,34 & 0,94 & 11,87 & 0,062 \\
\hline
\end{tabular}

Regressão logística múltipla.

\section{Discussão}

A prevalência de ansiedade em pacientes com STC foi de $28,7 \%$ e a prevalência de depressão foi de 37,6\%. O tabagismo e a renda familiar apresentaram associação estatisticamente significativa com a frequência de ansiedade dos pacientes com a STC $(p=0,035$ e $p=0,011$ respectivamente). A frequência de depressão apresentou associação estatisticamente significativa isoladamente com outras doenças e com a renda familiar dos pacientes $\operatorname{com} \operatorname{STC}(p=0,021$ e $p=0,028$ respectivamente).

Nosso estudo demonstrou que mulheres com a STC apresentaram risco de possuir ansiedade 18,55 vezes em relação aos homens. Acredita-se que as concentrações de estrogênio induzidas pelos inibidores da aromatase reduzem o efeito antinociceptivo do estrogênio, o que diminui o limiar para os estímulos dolorosos e, consequentemente, aumentando o risco de sintomas de afecções musculoesqueléticas, dentre elas a STC. ${ }^{11}$

Verificamos que ex-tabagistas apresentaram risco de ansiedade 6,05 vezes maior que pessoas não tabagistas. Em relação à renda familiar, a cada categoria de aumento da renda houve risco aumentado de ansiedade em 5,18 vezes, independente das demais características avaliadas.

Mulheres com a STC apresentaram risco de depressão 8,91 vezes maior que em homens. Ex-tabagistas apresentaram risco de depressão 4,30 vezes mais que naqueles não tabagistas e a cada aumento na categoria de renda familiar houve aumento de 4,26 vezes no risco de depressão independente das demais características dos pacientes.

Adesão a múltiplos estilos de vida saudáveis, índice de massa corporal dentro da normalidade, não fumantes, adequada atividade física e de lazer, alta ingestão de vegetais e frutas, e duração adequada do sono foi associado a um risco significativamente menor de depressão. ${ }^{12}$ Nosso estudo demonstrou que, apesar de não observarmos diferença estatisticamente signiciativa $(p>0,05)$ comparados isoladamente, o número de pessoas com sobrepeso ou obesidade ainda foi alto quando avaliados em conjunto, correspondendo a mais da metade da nossa amostra (66,3\%).

Nossos achados são consistentes com estudos anteriores, que mostram associação significativa entre alguns fatores do estilo de vida saudável combinados e sintomas depressivos. De acordo com Adjibade et al., ${ }^{12}$ pacientes com múltiplos fatores de estilo de vida saudável (não fumar, baixa ingestão de álcool, ser fisicamente ativo, ter dieta saudável e índice de massa corporal dentro da normalidade) apresentaram menor risco de sintomas de depressão em comparação com aqueles com apenas dois ou menos fatores de estilo de vida saudável. Em nossos resultados, o índice de massa corporal não foi correlacionado ao índice maior de depressão ou ansiedade quando comparados isoladamente; entretanto, quase dois terços das pessoas apresentavam-se com valores acima da normalidade. Em nosso estudo, tanto para ansiedade como para depressão, as características que influenciaram estatisticamente esses sintomas em pacientes com STC independente das demais características avaliadas foram gênero, tabagismo, e baixa renda familiar $(p<0,05)$. 


\section{Conclusão}

A prevalência de sintomas de ansiedade e depressão em pessoas com a STC é alta. $O$ gênero feminino, o tabagismo e a renda familiar foram as características da amostra que mais influenciaram na presença desses sintomas.

Conflito de Interesses

Os autores declaram não haver conflito de interesses.

\section{Referências}

1 Li Pi Shan R, Nicolle M, Chan M, et al. Electrodiagnostic Testing and Treatment for Carpal Tunnel Syndrome in Canada. Can J Neurol Sci 2016;43(01):178-182

2 World Health Organization. Depression and other common mental disorders: global health estimates. Disponível em: http://apps.who. int/iris/bitstream/handle/10665/254610/WHO-MSD-MER- 3032017. 2-eng.pdf?sequence $=1$. [Accessado em 20 de dezembro de 2018]

3 Doris A, Ebmeier K, Shajahan P. Depressive illness. Lancet 1999; 354(9187):1369-1375

4 Atroshi I, Gummesson C, Johnsson R, Ornstein E. Diagnostic properties of nerve conduction tests in population-based carpal tunnel syndrome. BMC Musculoskelet Disord 2003;4:9
5 Bakhshaie J, Zvolensky MJ, Goodwin RD. Cigarette smoking and the onset and persistence of depression among adults in the United States: 1994-2005. Compr Psychiatry 2015;60:142-148

6 Pereira-Miranda E, Costa PRF, Queiroz VAO, Pereira-Santos M, Santana MLP. Overweight and Obesity Associated with Higher Depression Prevalence in Adults: A Systematic Review and MetaAnalysis. J Am Coll Nutr 2017;36(03):223-233

7 Harvey SB, Modini M, Joyce S, et al. Can work make you mentally ill? A systematic meta-review of work-related risk factors for common mental health problems. Occup Environ Med 2017;74 (04):301-310

8 Nunez F, Vranceanu AM, Ring D. Determinants of pain in patients with carpal tunnel syndrome. Clin Orthop Relat Res 2010;468 (12):3328-3332

9 Kirkwood BR, Sterne JA. Essential medical statistics. 2nd ed. 2006. Massachusetts, USA: Blackwell Science; 2006

10 Hosmer DW, Lemeshow S. Applied Logistic Regression. 2nd ed. New York: Wiley; 2000

11 Al-Rousan T, Sparks JA, Pettinger M, et al. Menopausal hormone therapy and the incidence of carpal tunnel syndrome in postmenopausal women: Findings from the Women's Health Initiative. PLoS One 2018;13(12):e0207509

12 Adjibade M, Lemogne $C$, Julia C, et al. Prospective association between combined healthy lifestyles and risk of depressive symptoms in the French NutriNet-Santé cohort. J Affect Disord 2018;238:554-562 